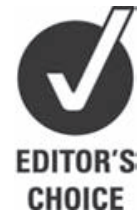

Correspondence to Dr Bruce B Forster, Department of Radiology, University of British Columbia and University of British Columbia, Koerner Pavilion, UBC Hospital, 2211 Wesbrook Mall, Vancouver, British Columbia V6T 2B5, Canada; bruce.forster@vch.ca

Accepted 18 August 2010 Published Online First 21 October 2010

\title{
Get a kick out of this: the spectrum of knee extensor mechanism injuries
}

\author{
Betty Tuong, Jeremy White, Luck Louis, Robyn Cairns, Gordon Andrews, \\ Bruce B Forster
}

\begin{abstract}
At the end of this article, the reader should be able to (1) recognise normal anatomy and anatomical variants of the extensor mechanism of the knee on various imaging modalities, including plain film, ultrasound and MRI; (2) diagnose a broad spectrum of EM injuries in adult and paediatric patients including patellar and quadriceps tendinopathy, Osgood-Schlatter disease, Sindig-Larsen-Johansson syndrome, chondromalacia patellae and patellar fractures on various imaging modalities; and (3) appreciate the important role of imaging in the diagnosis of musculoskeletal injuries.
\end{abstract}

\section{INTRODUCTION}

The extensor mechanism (EM) of the knee comprises the quadriceps muscles and tendon, the patella, patellar tendon and supporting retinaculum (figure 1). Injuries of the EM are common and may be due to acute trauma, overuse injuries or chronic degenerative disease, and imaging plays an important role in diagnosis. In addition to clinical evaluation, abnormalities of bone and soft tissues can be visualised with plain films and CT, and tendons can be assessed with ultrasound (US). MRI provides excellent evaluation of the knee and is often the imaging modality of choice in the characterisation of EM injuries.

\section{ANATOMY OF THE EM OF THE KNEE}

The EM of the knee comprises the quadriceps muscles and tendon, the patella, the patellar tendon and supporting retinacula. The quadriceps muscles include the rectus femoris and the vastus lateralis, vastus intermedius and vastus medialis. The origin of the rectus femoris is the anterior inferior iliac spine, and the vastus muscles originate from the femoral shaft. The quadriceps tendon inserts on the superior pole of the patella and usually has a trilaminar appearance on MR. ${ }^{1}$ The superficial lamina is composed of the rectus femoris, the intermediate lamina is composed of the vastus lateralis and medialis, and lastly the deep lamina is formed by the vastus intermedius.

The patella is the largest sesamoid bone in the body and articulates with the trochlear groove of the femur. It usually possesses a median ridge that divides the proximal patella into medial and lateral facets, and the medial facet is generally smaller. The patellar tendon is mainly composed of rectus femoris fibres that course over the patella and originates at the superior pole of the patella and inserts on the tibial tuberosity.

The medial retinaculum has three components: the superior component is composed of the vastus medialis and patellofemoral ligament, the midportion is composed of the medial collateral ligament fibres, and lastly the inferior component includes the patellotibial ligament and the medial patellomeniscal ligament. Similarly, the lateral retinaculum is composed of three parts: the superficial component includes the iliotibial tract and biceps femoris, the midportion is composed of the vastus lateralis, and the deep component is composed by the joint capsule.

Additional soft tissue structures includes fat pads, namely the anterior suprapatellar (quadriceps) fat pad, the posterior suprapatellar (prefemoral) fat pad and the infrapatellar or Hoffa's fat pad. The suprapatellar, pretibial and prepatellar bursae are also important structures.

Anatomy of the EM is best characterised on MR (figure 1). Plain films and CT provide excellent bony detail and soft tissue characterisation to a lesser degree. Tendons can be quickly and efficiently visualised on US and normally have an echogenic fibrillar appearance with parallel superficial and deep surfaces.

\section{IMAGING PITFALLS}

A common artefact in MRI called the "magic angle' phenomenon is unique to the musculoskeletal system, affecting tissues that are
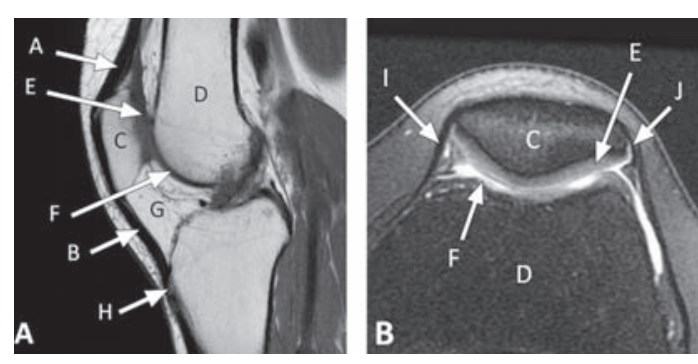

Figure 1 Normal MRI anatomy of the extensor mechanism of the knee in a 34-year-old male. $(A)$ Sagittal T1-weighted MR image: $A$, quadriceps tendon with normal striated appearance; $B$, patellar tendon; $C$, patella; $D$, femur; $E$, patellar cartilage; $F$, trochlear cartilage; $G$, Hoffa's fat pad; $H$, tibial insertion of the patellar tendon. (B) Axial proton density fat-saturated MR image labelled as above in (A), and I, medial retinaculum; J, lateral retinaculum. 

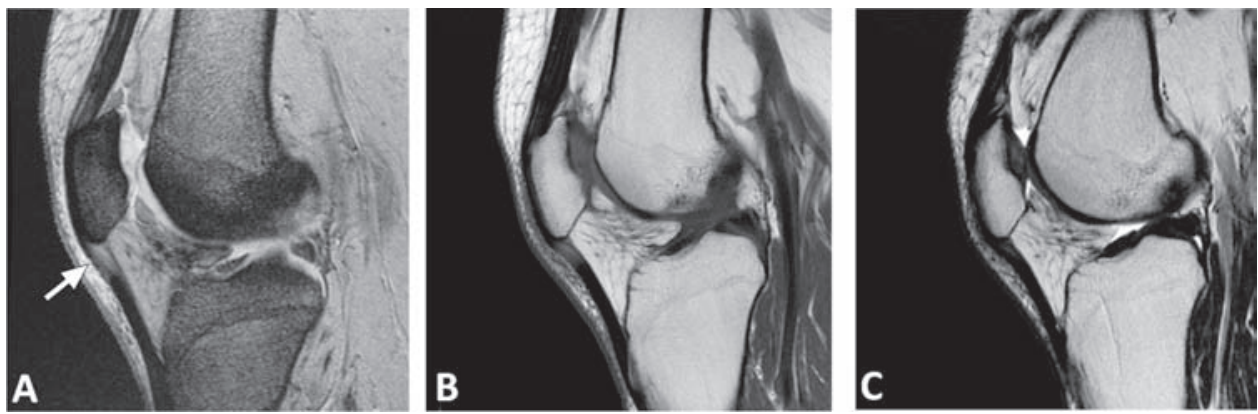

Figure 2 'Magic angle' phenomenon on an MR image of a 57-year-old female. (A) Sagittal gradient recalled echo sequence MR image in which the proximal portion of the patellar tendon appears hyperintense (arrow), an artefactual signal created when the tendon is angled at $55^{\circ}$ to the static magnetic field. (B) Sagittal T1-weighted MR image also demonstrating artefactual hyperintense signal at the proximal portion of the patellar tendon. (C) Sagittal T2-weighted MR image demonstrating reduction in the magic angle artefact seen on gradient recalled echo and T1 weighted MR images due to a longer echo time (87 ms).

composed of well-ordered collagen fibres, such as hyaline cartilage, menisci and tendons. These tissues appear to behave in an anisotropic fashion when placed in a magnetic field and appear hyperintense when the fibres are oriented at a 'magic angle' of approximately $55^{\circ}$ to the static field of the magnet. ${ }^{2}$ Thus, the artefact can be mistaken for the appearance of pathology (ie, tendinopathy) (figure 2). This artefact is most apparent at short echo times and can be reduced with an echo time of $37 \mathrm{~ms}$ or more (ie, T2-weighted sequences). ${ }^{2}$

On US, tendons also display an anisotropic effect when the probe is not oriented perpendicular to the tendon at $90^{\circ}$. As a result, areas of low echogenicity can be mistaken for tendinopathy. To overcome this pitfall, the probe is angled through its full range, and if the previously low echogenicity area within the tendon becomes more echoic and eventually isoechoic to the rest of the tendon, then tendon disease can be excluded. ${ }^{3}$

\section{ANATOMICAL VARIANTS}

\section{Articular muscle of the knee (articularis genu $\mathrm{m}$.)}

This muscle supports the suprapatellar bursa and the superior joint capsule of the knee. It originates from the anterior aspect of the distal femur and extends inferomedially to insert near the suprapatellar bursa (figure 3). In a 1995 retrospective study, the articular muscle of the knee could be seen in $83 \%$ of clinical MRI studies of the knee. ${ }^{4}$ It should not be mistaken for a soft tissue mass.

\section{Dorsal defect of the patella}

Dorsal defect of the patella (DDP) is an anomaly of ossification and is of little clinical significance. The characteristic lesion is round and lytic with well-defined margins and located in the superolateral aspect of the patella adjacent to the subchondral bone (figure 4).

\section{Bipartite patella}

This anatomical variant is a result of failure of an accessory ossification centre to unite with the main body of the patella. As a result, the accessory ossification centre unites with the patella via a synchondrosis. The most common location for the secondary ossification centre is at the superolateral aspect of the patella and can be mistaken for a patellar fracture. It can be differentiated both by its position
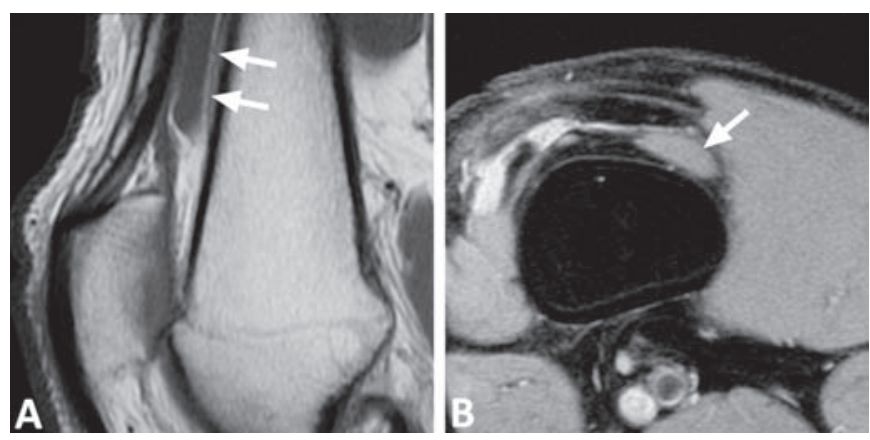

Figure 3 Articular muscle of the knee in a 42-year-old female.

Sagittal T1-weighted (A) and axial proton density fat saturated (B) MR images demonstrating an accessory muscle (arrows) independent from the vastus muscle group which extends from the anterior surface of the femur to insert on the suprapatellar bursa.

and the smooth, corticated margins along adjoining surfaces (figure 5).

\section{PATHOLOGY}

\section{Patellar tendinopathy}

Patellar tendinopathy or 'jumper's knee' is a common overuse condition that classically occurs in young athletes who jump, kick and run, placing repeated stress on the patellofemoral joint. Patellar tendinopathy includes a spectrum of pathology from chronic degeneration to partial tearing. Lesions in patellar tendinopathy typically occur in the deep posterior portion of the proximal patellar tendon adjacent to the lower pole of the patella. On US, the normal fibrillar pattern of the patellar tendon is disrupted with decreased echogenicity and thickening near its proximal insertion (figure 6A)..$^{5}$ Increased flow on colour Doppler indicating neovascularisation can also help localise the site of injury (figure 6B). ${ }^{6}$ Typical findings on MRI include focal thickening of the proximal one-third of the patellar tendon with abnormal high signal intensity on T2-weighted images (figures 6C and $6 \mathrm{D}){ }^{7}$

\section{Patellar tendon rupture}

Rupture of the patellar tendon is rare, but the most common site of rupture is at the junction of the tendon with the lower pole of the patella. It is usually caused by violent contraction of the quadriceps muscle against the fixed load of the 

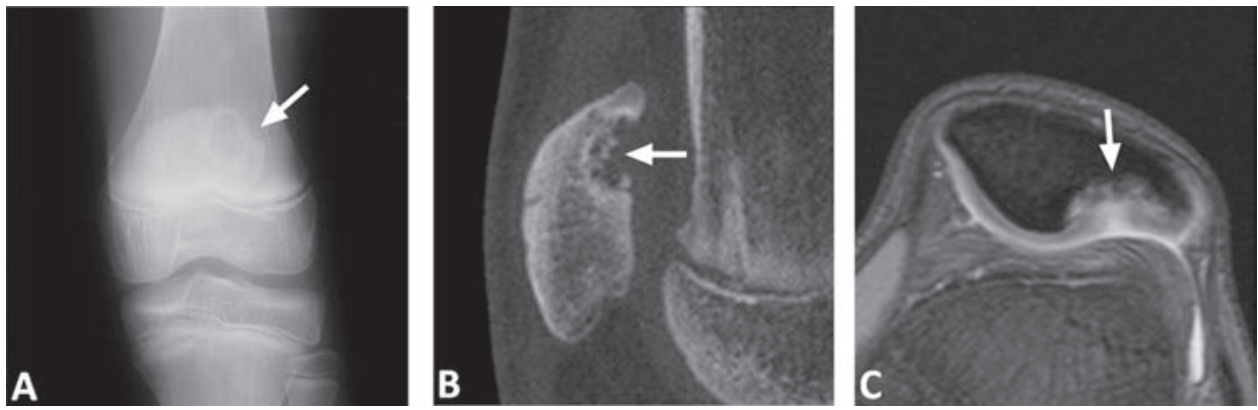

Figure 4 Dorsal defect of the patella in a 12-year-old male. Anteroposterior radiograph of the knee (A) and a reconstructed sagittal CT image (B) shows a well-defined, round and lytic lesion in the superolateral aspect of the patella adjacent to the subchondral bone (arrow). Axial gradient recalled echo MR image $(C)$ demonstrates a defect in the superolateral aspect of the patella with intact overlying cartilage that is mildly thickened with increased signal (arrow).
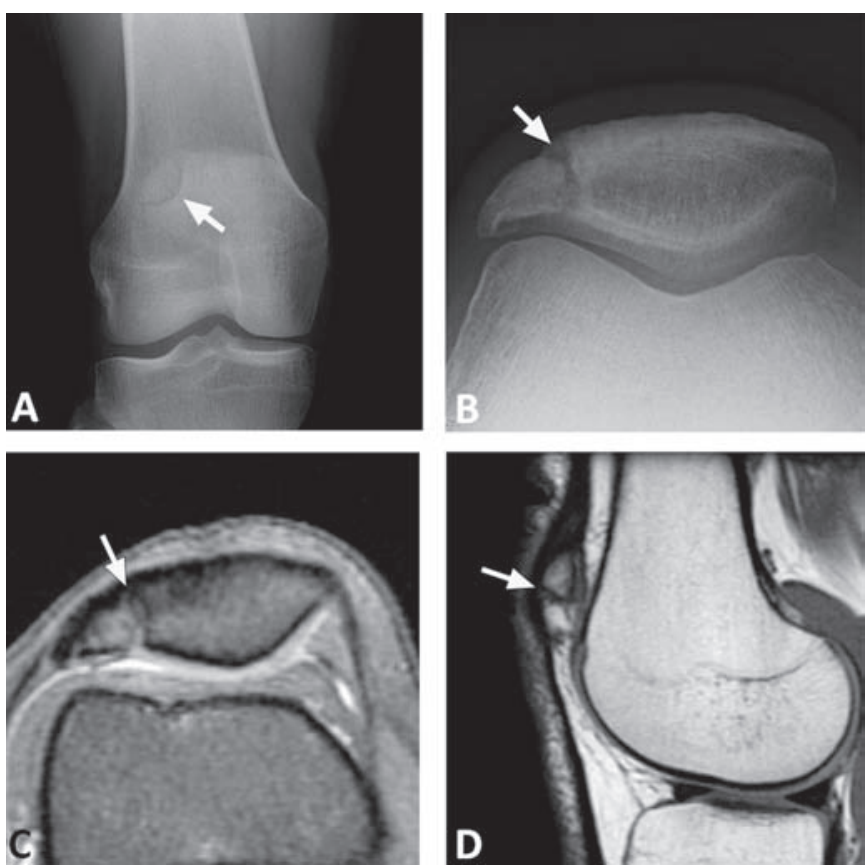

Figure 5 Bipartite patella in a 42-year-old woman with lateral knee pain. Anteroposterior $(A)$ and skyline $(B)$ radiographs of the knee demonstrate an osseous fragment in the superolateral aspect of the patella with smooth, corticated margins (arrow). Axial proton density fat saturated (C) and sagittal T1-weighted (D) MR images demonstrate cartilage overlying both osseous parts of the patella.

patient's body weight with the knee in flexion. ${ }^{8}$ The injury is suggested on radiographs by a superiorly displaced patella and infrapatellar soft tissue swelling (figure 7A). On MR, the patellar tendon is completely torn from its patellar attachment and can appear buckled (figure 7B).

\section{Osgood-Schlatter disease}

Osgood-Schlatter disease (or tibial tubercle apophyseal injury) is a common cause of knee pain in adolescents. It is caused by repeated traction on the immature tibial tuberosity by the patellar tendon, which can cause inflammation, avulsion fractures and excess bone growth. Osgood-Schlatter disease is usually a clinical diagnosis, and imaging is used to rule out other pathologies. The diagnosis of Osgood-Schlatter disease can only be suggested on plain films if radiographic soft tissue swelling is present anterior to the tibial apophysis
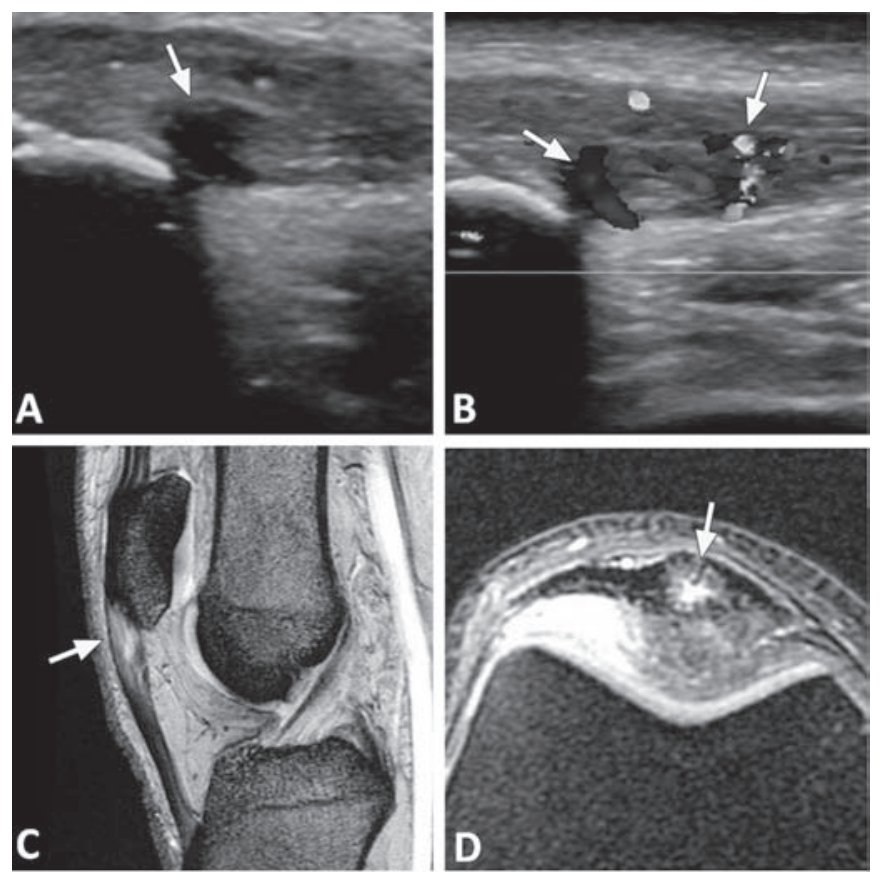

Figure 6 Patellar tendinopathy in a 34-year-old male with anterior knee pain. (A) Longitudinal sonogram of the patellar tendon showing fusiform swelling of the proximal patellar tendon and a hypoechoic area effacing the fibrillar appearance of the tendon (arrow). (B) Colour Doppler sonogram (in greyscale) of the patellar tendon demonstrating the same findings as in $(A)$ with increased vascularity on Doppler (arrows). (C) Sagittal gradient recalled echo MR image demonstrating thickening of the proximal patellar tendon and internal high signal intensity (arrow). (D) Axial proton density fat saturated MR image showing high signal at the attachment of the patellar tendon to the patella (arrow).

(figure 8), as fragmentation of the tibial apophysis can be a normal variant.

\section{Sindig-Larsen-Johansson syndrome}

This syndrome is caused by traction on the patellar tendon, causing inflammation at the insertion of the proximal tendon into the inferior pole of the patella. It usually appears in children after a period of rapid growth and can be distinguished from patellar tendinopathy by the presence of bone marrow oedema in the patella (figure 9), and by clinical presentation. 

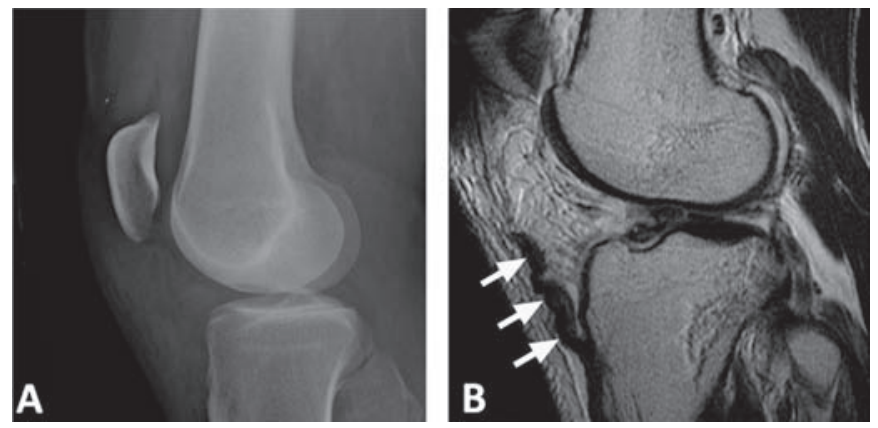

Figure 7 Patellar tendon rupture in a 32-year-old male who sustained trauma to the knee while dirt bike riding. (A) Lateral radiograph of the knee demonstrating ill-definition of the proximal aspect of the patellar tendon with infrapatellar soft tissue swelling. A metallic foreign body from prior surgery (repair of a right tibial plateau fracture requiring open reduction internal fixation and subsequent hardware removal) is seen in the superficial suprapatellar soft tissue. (B) Oblique sagittal T2-weighted fast spin echo MR image showing a buckled retracted patellar tendon (arrows), completely torn from its patellar attachment. Note the susceptibility artefact from prior surgery as described in (A).

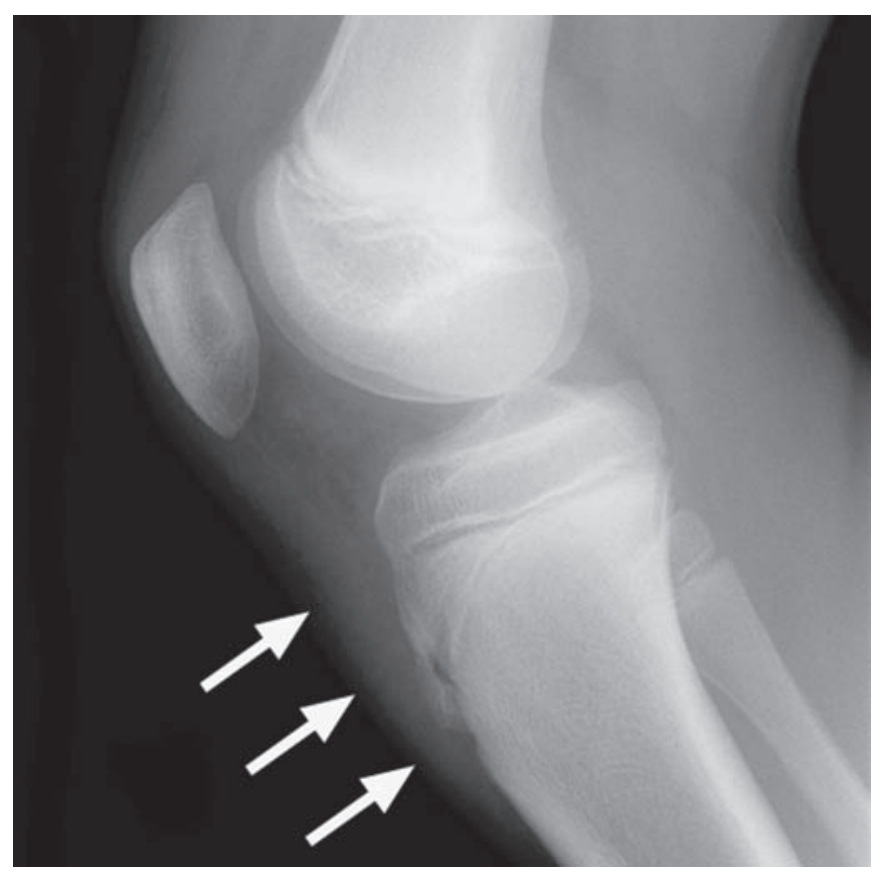

Figure 8 Osgood-Schlatter disease in an 11-year-old female with anterior knee pain. Lateral radiograph of the knee demonstrates soft tissue swelling anterior to the tibial tuberosity (arrows).

\section{Quadriceps tendinopathy}

Quadriceps tendinopathy occurs less frequently than patellar tendinopathy but demonstrates similar findings on imaging (figure 10) ${ }^{9}$. The most common site of injury is at the distal site of insertion on the patella. Tears of the quadriceps tendon usually occur in an older patient population, and a predisposing risk factor, such as diabetes, gout or rheumatoid arthritis, often exists. In the younger patient, acute tears can be due to forced muscle contraction or blunt trauma.

\section{Quadriceps tendon rupture}

Rupture of the quadriceps tendon is relatively infrequent and is more common in patients older than 40 years ${ }^{10}$. It

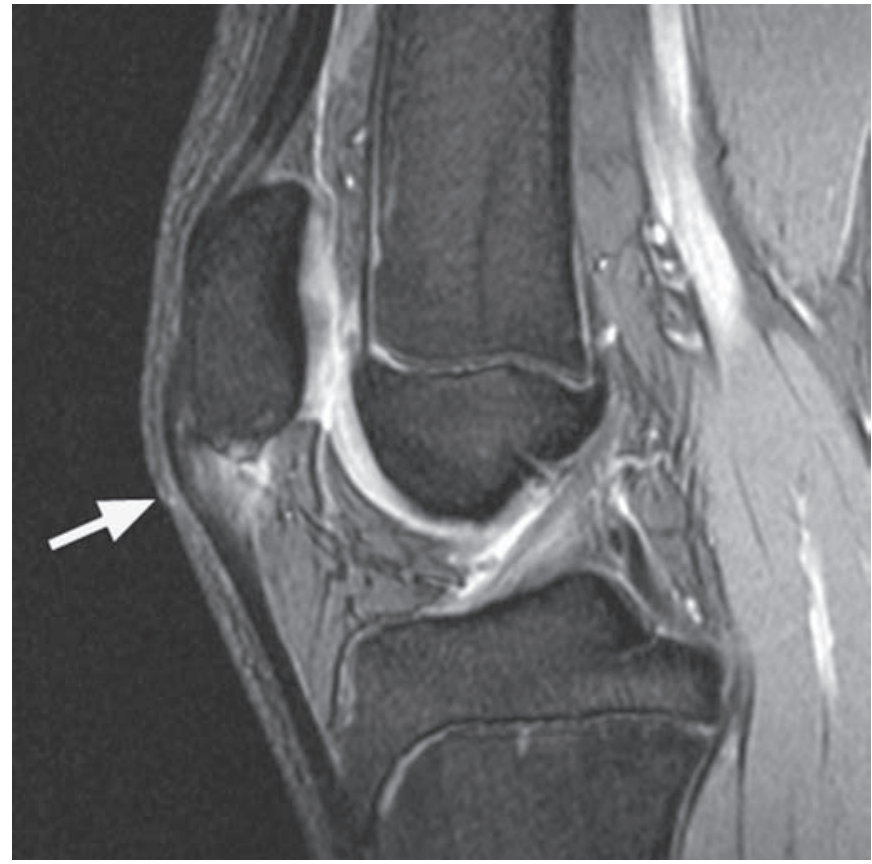

Figure 9 Sindig-Larsen-Johansson syndrome in a paediatric patient. Sagittal gradient recalled echo MR image demonstrates thickening and increased signal of the proximal patellar tendon (arrow) and irregular ossification of the inferior pole of the patella with underlying bone marrow oedema.
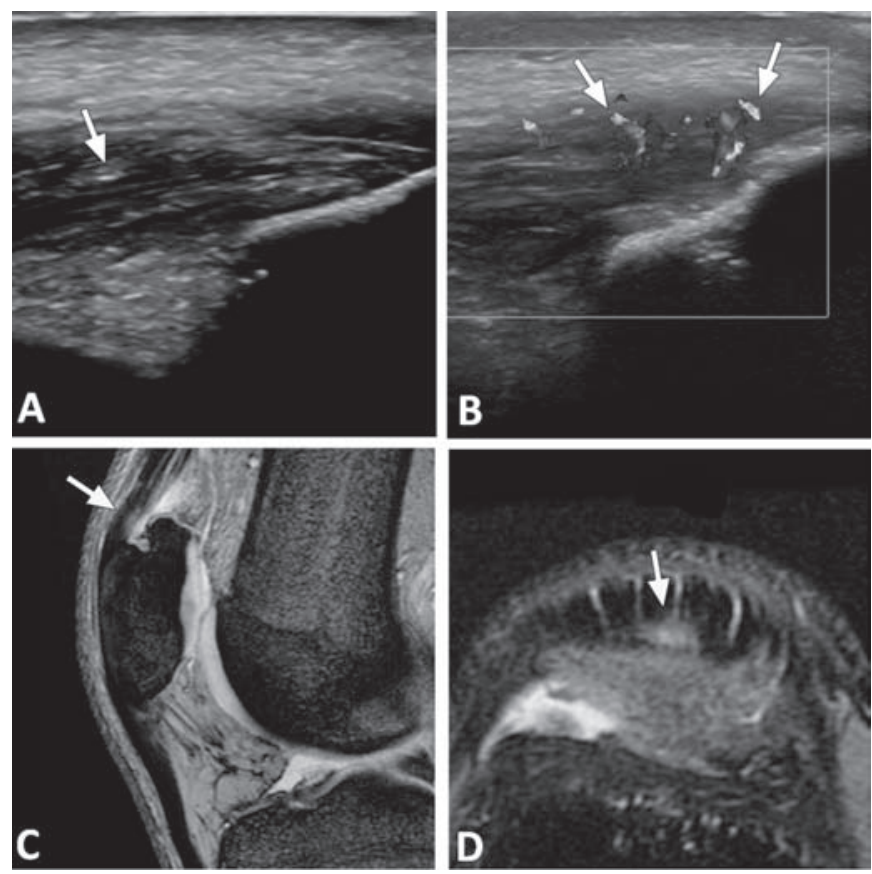

Figure 10 Quadriceps tendinopathy in a 26-year-old male professional volleyball player with suprapatellar knee pain. (A) Longitudinal sonogram demonstrates dystrophic calcification (arrow) within the distal quadriceps tendon and multiple hypoechoic areas effacing the fibrillar appearance of the tendon. (B) Colour Doppler sonogram (in greyscale) showing the same findings as in $(A)$, with increased vascularity (arrows). (C) Sagittal gradient recalled echo MR image showing thickening and increased signal of the distal quadriceps tendon at its insertion (arrow). (D) Axial proton density fat saturated MR image showing high signal at the attachment of the quadriceps tendon to the patella (arrow). 


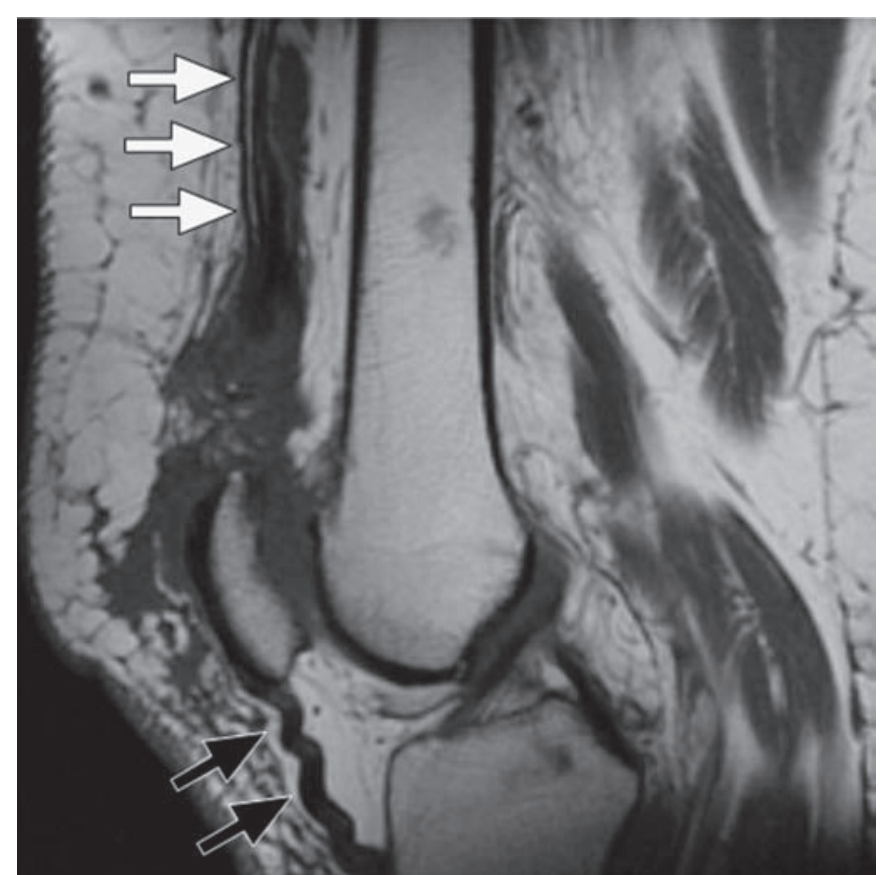

Figure 11 Quadriceps tendon rupture in a 53-year-old female who fell. Sagittal T1-weighted MR image shows a complete tear of the quadriceps tendon at the superior pole of the patella. There is 3 $\mathrm{cm}$ retraction of the quadriceps tendon (white arrows), as well as 'wrinkling' of the intact patellar tendon (black arrows) which is no longer under tension. usually occurs in the setting of underlying disease, including chronic renal failure, diabetes, rheumatoid arthritis, gout and obesity. Most ruptures of the quadriceps tendon occur during attempts to regain balance to avoid a fall. The quadriceps muscle rapidly contracts against the patient's body weight (eccentric contraction) while the knee is in a semiflexed position, placing the quadriceps tendon under its greatest tensile stress. ${ }^{10}$ Findings on MR include complete rupture of the quadriceps tendon with a 'wrinkled' appearance of the patellar tendon, which no longer has a tensile force applied to it (fig 11). ${ }^{11}$

\section{Patellar fractures}

Patellar fractures are usually sustained through blunt trauma or forced contraction and result in transverse or stellate fractures. They can be mistaken for bipartite patella (figure 5) but can be distinguished from this anatomical variant based on orientation and the presence of indistinct margins and noncorticated irregular fragments (figure 12). While plain films can detect the presence of fractures, they are better delineated with CT.

In paediatric patients, osteochondral sleeve fractures are the most common patellar fractures, although they are rare. ${ }^{12}$ A full circumference of cartilaginous tissue and often a bony fragment is avulsed from the lower pole of the patella due to rapid and forceful muscle contraction against a partially flexed knee. This occurs most often with jumping, and skateboarding is a common cause. ${ }^{12}$ On plain films, an abnormally superiorly displaced patella and/or a small bone fragment distal to the lower pole of the patella can provide a clue to
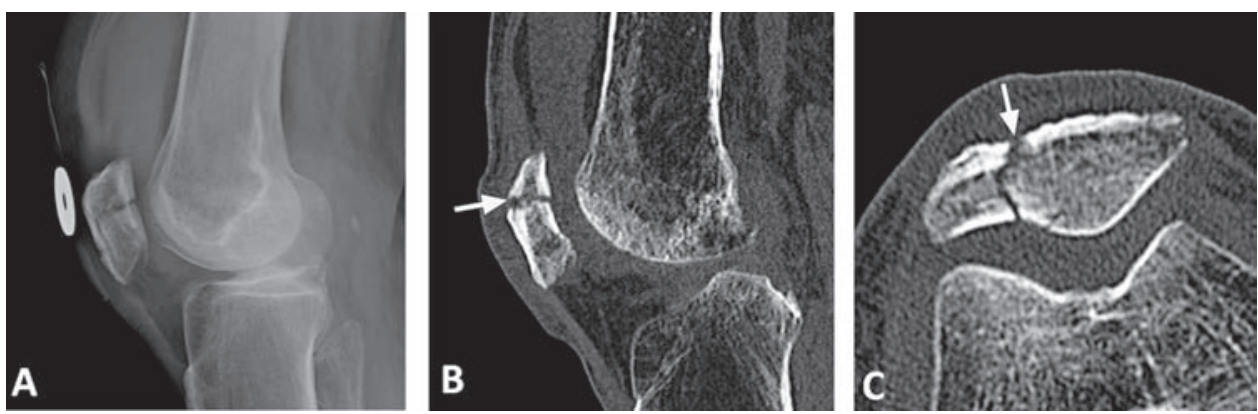

Figure 12 Patellar fracture in a 78-year-old female who fell. (A) Lateral radiograph of the knee demonstrating a multiple linear lucencies in keeping with a stellate fracture of the patella with surrounding soft tissue swelling. (B) Reconstructed sagittal CT image showing a horizontally oriented linear fracture of the patella (arrow). (C) Axial CT image demonstrating a vertically oriented linear patellar fracture (arrow).
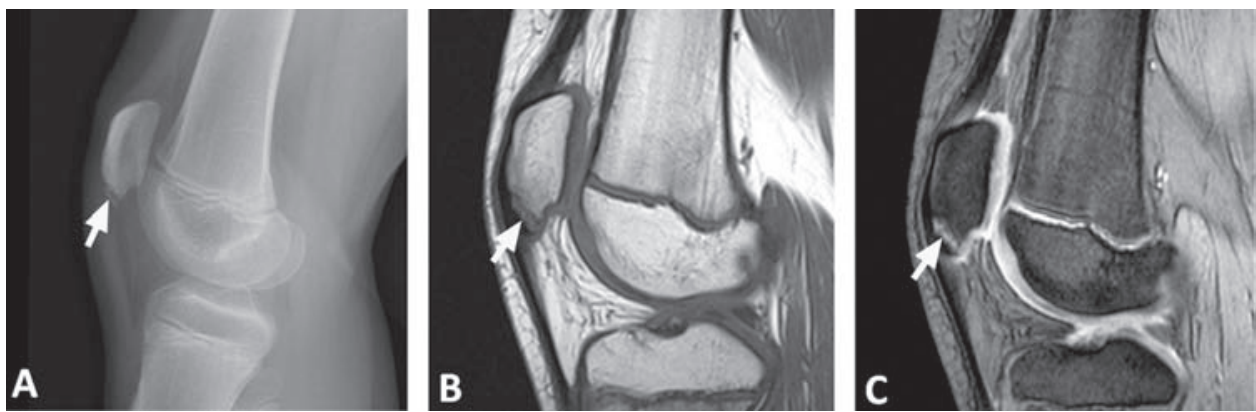

Figure 13 Osteochondral sleeve fracture in a paediatric patient. (A) Lateral plain film of a patellar avulsion fracture of the inferior pole of the patella demonstrating a superiorly displaced patella and a small bone fragment (arrow). Sagittal T1-weighted (B) and sagittal gradient recalled echo (C) MR images demonstrating an osteochondral fracture of high signal intensity with a small bone fragment (arrow). 


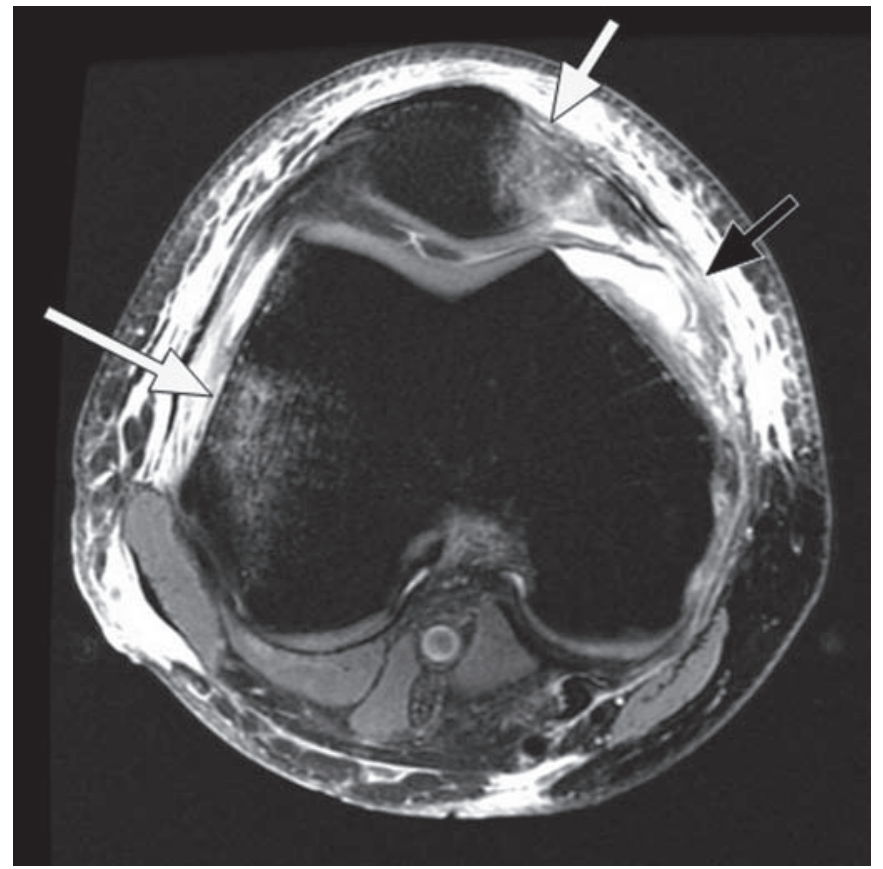

Figure 14 Lateral patellar dislocation/relocation in a 25-year-old male who sustained trauma to the knee. Axial proton density fat saturated MR image demonstrates the triad of findings in lateral patellar dislocation/relocation: subchondral oedema in the medial facet of the patella (short white arrow) and in the anterolateral femoral condyle (long white arrow), and a tear in the medial retinaculum with contour irregularity and surrounding high signal intensity oedema (black arrow).

Table 1 Shahriaree arthroscopic grading system for chondromalacia patellae and the corresponding theoretical MRI findings ${ }^{17}$

\begin{tabular}{|c|c|}
\hline Shahriaree arthroscopic stage & MRI findings \\
\hline $\begin{array}{l}\text { I-Softening and swelling of articular } \\
\text { cartilage }\end{array}$ & $\begin{array}{l}\text { Focal signal intensity changes without } \\
\text { contour deformity (difficult to assess } \\
\text { on MRI) }\end{array}$ \\
\hline $\begin{array}{l}\text { II-Blistering of cartilage with intact } \\
\text { surface }\end{array}$ & $\begin{array}{l}\text { Focal signal intensity change and contour } \\
\text { bulge (partial thickness) }\end{array}$ \\
\hline $\begin{array}{l}\text { III-Surface irregularity/ulceration/ } \\
\text { fibrillation (not extending down to the } \\
\text { bone) }\end{array}$ & $\begin{array}{l}\text { Focal signal intensity change, contour } \\
\text { irregularities, cartilage thinning and fluid } \\
\text { extension into cartilage (full thickness) }\end{array}$ \\
\hline $\begin{array}{l}\text { IV-Similar to stage III, but the } \\
\text { ulceration extends to the subchondral } \\
\text { bone ( } \pm \text { subchondral bony changes) }\end{array}$ & $\begin{array}{l}\text { Similar to stage III with defects extending } \\
\text { to the cortical bone (with subchondral } \\
\text { bony changes) }\end{array}$ \\
\hline
\end{tabular}

diagnosis. On MR, cartilaginous injury can be easily detected due to the contrast between hypointense cartilage and the hyperintense fracture line. It can also delineate the extent of cartilaginous injury in further detail and define the relationship of the fracture fragments to help in further management (figure 13). ${ }^{13}$

\section{Patellar dislocation/relocation}

Patellar dislocation most commonly results from a twisting motion and occurs in a lateral direction. The natural history for most patellar dislocations is for the patella to relocate prior to the patient's presentation, thereby confusing the clinical picture. Lateral patellar dislocation/relocation results in demonstratable injury to both soft tissue (retinacular disruption) and bony structures. The medial patellar facet impacts against the lateral femoral condyle, which is seen on MRI images as bone marrow contusion or fracture, findings pathognomonic for patellar dislocation. ${ }^{14} \mathrm{~A}$ distinct triad of findings can be seen on MRI (figure 14), including subchondral oedema in the medial facet of the patella and in the anterolateral femoral condyle with a tear in the medial retinaculum. In children, patellar dislocation/relocation often results in an osteochondral fracture and can result in large intra-articular loose fragments.

\section{Chondromalacia patellae}

Chondromalacia patellae (also known as patellofemoral syndrome) is a common cause of anterior knee pain especially in athletes due to degradation of the patellar hyaline cartilage. It is difficult to diagnose clinically. The gold standard for chondral assessment is arthroscopy, and a widely used four-stage grading system of pathological change was developed by Shahriaree. ${ }^{15}$ However, MRI can accurately assess the condition of the articular cartilage for more severe grades of chondromalacia patellae (table 1, figure 15) and is a reliable diagnostic tool for investigation of anterior knee pain. ${ }^{16}$

\section{Prepatellar bursitis}

The prepatellar bursa is a synovial lined sac containing synovial fluid located anterior to the patella and below the skin. Prepatellar bursitis or 'housemaid's knee' may result from a variety of causes, including acute trauma, chronic friction or repetitive minor injuries (such as repeated kneeling),

Figure 15 Chondromalacia patellae grades II-IV in various patients. (A) Axial fast spin echo proton density fat saturated (FSE PD FS) MR image of chondromalacia patellae grade II in a 46-year-old male. High signal is seen in the patellar cartilage in the lateral patellar facet (arrows). (B) Axial FSE PD FS MR image of chondromalacia patellae grade III in a 51-year-old female. There is full thickness focal signal intensity change, contour irregularity and thinning of the cartilage (arrows). (C) Axial PD MR image of chondromalacia patellae grade IV in a 63-year-old female. There is extensive full-thickness chondral loss along the medial patellar facet. There is also associated marrow oedema and cystic change within the

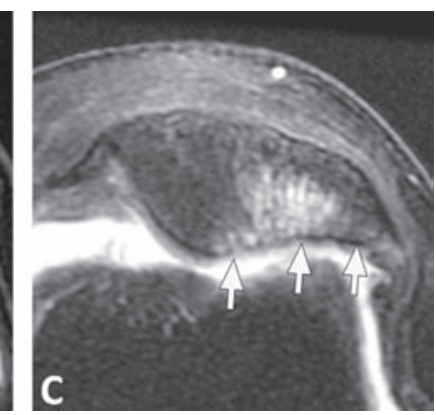
adjacent bone of the patella (arrows). 

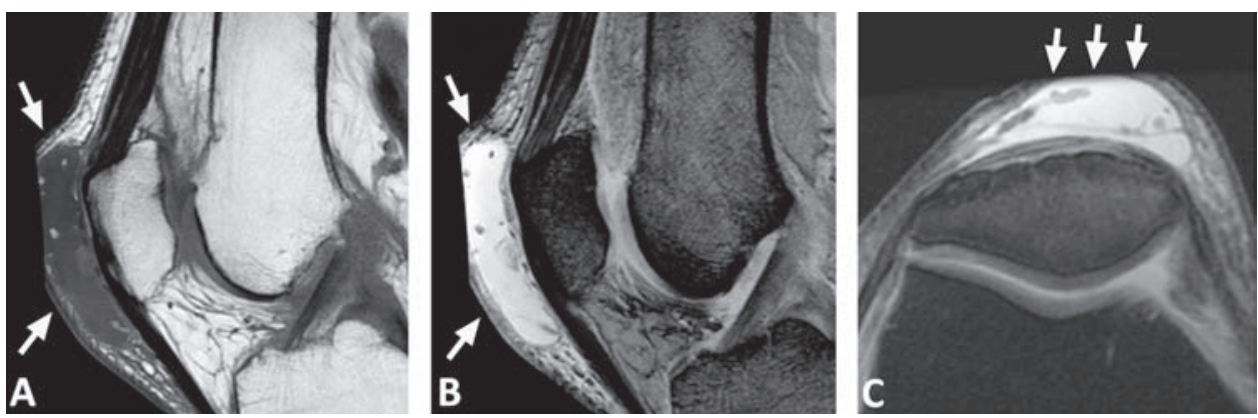

Figure 16 Prepatellar bursitis. Sagittal T1-weighted (A), sagittal gradient recalled echo (B) and axial fast spin echo proton density fat saturated MR images (C) of prepatellar bursitis demonstrate a complex fluid collection anterior to the knee in the region of the prepatellar bursa (arrows). The patient's knee is markedly enlarged, and thus falls outside the field of view.

inflammation, crystal deposition (as in gout or pseudogout) or infection (through direct inoculation or haematogeneous spread). Findings on MRI may include a complex fluid collection anterior to the knee in the region of the prepatellar bursa (figure 16), and the wall of the bursa may be thickened and irregular.

\section{CONCLUSION}

In addition to clinical evaluation, a wide range of imaging modalities, including plain film, CT, US and MRI, play a major role in the diagnosis of a variety of EM injuries.

Competing interests None.

Provenance and peer review Not commissioned; externally peer reviewed.

\section{REFERENCES}

1. Zeiss J, Saddemi SR, Ebraheim NA. MR imaging of the quadriceps tendon: normal layered configuration and its importance in cases of tendon rupture. Am J Roentgenol 1992;159:1031-4.

2. Peh WC, Chan JH. Artifacts in musculoskeletal magnetic resonance imaging: identification and correction. Skeletal Radiol 2001;30:179-91.

3. Connolly DJ, Berman L, McNally EG. The use of beam angulation to overcome anisotropy when viewing human tendon with high frequency linear array ultrasound. Br J Radiol 2001:74:183-5.

4. Puig S, Dupuy DE, Sarmiento A, et al. Articular muscle of the knee: a muscle seldom recognized on MR imaging. Am J Roentgenol 1996:166:1057-60.
5. Khan KM, Bonar F, Desmond PM, et al. Patellar tendinosis (jumper's knee): findings at histopathologic examination, US, and MR imaging. Victorian Institute of Sport Tendon Study Group. Radiology 1996;200:821-7.

6. Weinberg EP, Adams MJ, Hollenberg GM. Color Doppler sonography of patellar tendinosis. Am J Roentgenol 1998;171:743-4.

7. Yu JS, Popp JE, Kaeding CC, et al. Correlation of MR imaging and pathologic findings in athletes undergoing surgery for chronic patellar tendinitis. Am J Roentgeno/ 1995:165:115-18.

8. Matava MJ. Patellar tendon ruptures. J Am Acad Orthop Surg 1996:4:287-96.

9. Pfirrmann CW, Jost B, Pirkl C, et al. Quadriceps tendinosis and patellar tendinosis in professional beach volleyball players: sonographic findings in correlation with clinical symptoms. Eur Radiol 2008;18:1703-9.

10. Ilan DI, Tejwani N, Keschner M, et al. Quadriceps tendon rupture. J Am Acad Orthop Surg 2003:11:192-200.

11. Berlin RC, Levinsohn EM, Chrisman $\mathrm{H}$. The wrinkled patellar tendon: an indication of abnormality in the extensor mechanism of the knee. Skeletal Radiol 1991;20:181-5.

12. Hunt DM, Somashekar N. A review of sleeve fractures of the patella in children. Knee 2005;12:3-7.

13. Bates DG, Hresko MT, Jaramillo D. Patellar sleeve fracture: demonstration with MR imaging. Radiology 1994;193:825-7.

14. Kirsch MD, Fitzgerald SW, Friedman H, et al. Transient lateral patellar dislocation: diagnosis with MR imaging. Am J Roentgenol 1993;161:109-13.

15. Shahriaree H. Chondromalacia. Contemp Orthop 1985;11:27-39.

16. Pihlajamäki HK, Kuikka PI, Leppänen VV, et al. Reliability of clinical findings and magnetic resonance imaging for the diagnosis of chondromalacia patellae. $J$ Bone Joint Surg Am 2010;92:927-34.

17. Scuderi GR, (Ed) . The Patella (first edition). New York: Springer Verlag, 1995:101. 